\title{
The Effect of Biofilm-Forming Bacteria on Inflammasome Activity
}

Georgia Lea Williams, BS, Suman Santra, PhD, Sashwati Roy, PhD

Indiana University School of Medicine

Indiana Center for Regenerative Medicine \& Engineering

Department of Surgery

Background and Hypothesis: Over $75 \%$ of chronic wounds have biofilm infection. Biofilm infection derails the overall process of wound healing. Biofilm bacteria have developed strategies to subvert macrophage inflammatory response. The underlying mechanisms of such subversion are currently unknown. Inflammasomes are multiprotein, intracellular complexes that contain caspase-1. Caspase-1 is responsible for cleavage and activation of cytokines IL$1 \beta$, a major pro-inflammatory cytokine in chronic wounds. We hypothesized that biofilm bacteria subvert macrophage inflammatory response by attenuating caspase- 1 activity and thereby reducing IL-1 $\beta$. The primary objective of the study was to determine Caspase- 1 activity and IL- $1 \beta$ production by blood monocytes derived from patient blood samples exposed to biofilm infection ex vivo and clinical wound macrophages.

\section{Methods:}

Wound macrophages were isolated from chronic patients seen at Indiana University Health Comprehensive Wound Center (CWC). Blood monocyte derived macrophages (BMDMs) were exposed to conditioning media from isogenic mutant strains SA300 $\Delta$ sarA or SA300 $\Delta$ rexB (derived from Staphylococcus aureus USA300LAC) as hypo- and hyper-biofilm forming mutants. Wound macrophages were isolated from wound fluid with known bacterial content. The IL- $\beta$ release and caspase- 1 activity from macrophages were determined using ELISA, PCR, and a colorimetric assay.

\section{Results:}

Both RT-PCR and ELISA independently exhibited a significant reduction in IL-1 $\beta$ production in macrophages exposed to conditioned media from hyper-biofilm forming SA300 $\Delta$ rexB as compared to hypo-biofilm forming SA300 $\Delta$ sarA mutant. The Caspase-1 activity was significantly reduced in the macrophages challenged with hyper-biofilm forming bacteria as compared to hypo-biofilm forming mutant.

\section{Conclusion and Potential Impact:}

Biofilm forming bacteria leads to an attenuation in production of IL-1 $\beta$ from macrophages indicating a subversion of inflammatory response. The caspase-1 activity data exhibited a significant role of caspase-1 in biofilm mediated subversion of the inflammatory response indicating a reduction in inflammasome activity. A clear understanding of the mechanisms of biofilm mediated suppression of host response will help improve therapeutic strategies against wound biofilm infection. 